\title{
Three ways to lattice Boltzmann: a unified time-marching picture
}

\author{
S. Ubertini ${ }^{1}$, P. Asinari ${ }^{2}$, S. Succi ${ }^{3}$ \\ ${ }^{1}$ Università di Napoli "Parthenope", \\ DiT - Dipartimento per le Tecnologie, \\ Centro Direzionale, Is. C4, 80143 Napoli, Italy \\ ${ }^{2}$ Politecnico di Torino, Dipartimento di Energetica, \\ Corso Duca degli Abruzzi 24, 10129, Torino, Italy \\ 3 Istituto Applicazioni Calcolo, CNR, \\ V.le del Policlinico 137, 00161, Roma, Italy
}

(Dated: November 9, 2009)

\begin{abstract}
It is shown that the lattice Boltzmann equation (LBE) corresponds to an explicit, Verlet timemarching scheme for a continuum generalized Boltzmann equation with a memory delay equal to a half time-step. This proves second-order accuracy of LBE with respect to this generalized equation, with no need of resorting to any implicit time-marching procedure (Crank-Nicholson) and associated non-linear variable transformations ([1]). It is also shown, and numerically demonstrated, that this equivalence is not only formal, but translates into a complete equivalence of the corresponding computational schemes with respect to the hydrodynamic equations. Second order accuracy with respect to the continuum kinetic equation is also numerically demonstrated for the case of the Taylor-Green vortex. It is pointed out that the equivalence is however broken for the case in which mass and/or momentum are not conserved, such as for chemically reactive flows and mixtures. For such flows, the time-centered implicit formulation may indeed offer a better numerical accuracy.
\end{abstract}

PACS numbers: 47.11.-j, 47.65.Cb 


\section{INTRODUCTION}

In the last decade the lattice Boltzmann (LB) method has emerged as a competitive technique for the numerical simulation of complex flows ([2-6]) and, to a much lesser extent, even quantum systems ([7]). Viewed as a pure hydrodynamic solver, LB exhibits a number of remarkable properties, which stem from the conceptual simplicity of the stream-andcollide mathematical structure inherent to kinetic theory, as opposed to the macroscopic representation of fluid motion. From the accuracy stand-point LB, can be classified as a second-order method with regards to the spatial discretization, whose efficiency results from a low computational demand (operation count per lattice site and grid point) as combined with exact conservation for the streaming step and machine round-off conservation for the collision stage. These conservation laws are instrumental in keeping the prefactors of higher order errors much lower than in finite-difference schemes, thus allowing LB to compete with higher-order schemes, and even spectral methods. The time accuracy of the LB method has made the object of some controversy in the past $([1,8-10])$; indeed, being based on a forward-Euler time marching scheme, it would seem natural to conclude that LB is only first order accurate in time, the common tenet being that second order accuracy is achieved by absorbing second-order numerical diffusivity into the effective fluid viscosity. Only recently it was realized that a simple time marching formulation of the LB method based on an implicit Crank-Nicholson (CN) integration rule can achieve second-order time accuracy as shown by [1]. This approach, however, requires a non-linear transformation of the LB discrete distribution to an equivalent one, based on a recombination of equilibrium and nonequilibrium components. Since these transformation is one-to-one, it was concluded that the $\mathrm{CN}$ version of LB is indeed second-order in time. In this paper we wish to bring up the following observations. i) The standard and implicit CN versions of LB are formally and computationally equivalent, (provided a time shift $\Delta t / 2$ is retained in the relaxation time of the explicit version). ii) The LB can also be derived as a second-order explicit (Verlet) scheme for a continuum Boltzmann equation with a memory delay term exactly equal to $\Delta t / 2$. iii) All of these schemes are second order in space and first order in time with respect to hydrodynamic limit (diffusive scaling). iv) All of these schemes are second order in space and time with respect to the continuum kinetic limit (convective scaling). v) The equivalence between these three independent formulations only applies to the case of flows with mass 
and momentum conservation. For more general situations, e.g. flow mixtures with chemical reactions, the implicit $\mathrm{CN}$ formulation may indeed offer an advantage over the standard one.

\section{STANDARD AND GENERALISED BOLTZMANN EQUATION WITH MEMORY}

The Boltzmann equation (BE) rests on a clearcut separation between free-streaming and collisions, which results in a very compact first-order hyperbolic equation:

$$
D f=\omega\left(f^{e q}-f\right)
$$

where $D:=\partial_{t}+v_{a} \partial_{a} f$ is the free-streaming operator, and the collision operator is taken in the form of a single-time relaxation around a local Maxwellian equilibrium, $f^{e q}, \omega$ being the inverse relaxation time, i.e. $\omega=1 / \tau$. Finally, Latin indices run over spatial dimensions.

The gas-kinetic equation $([11,12])$ (BGK model for the Boltzmann equation) provides a useful mathematical model for fluid dynamics in the limit of weak departure from local equilibrium (hydrodynamic regime).

The fluid mass density and velocity are defined by the zeroth and first order kinetic moments of the distribution, that is $\rho(\vec{x}, t)=\int f(\vec{x}, \vec{v}, t) d \vec{v}$ and $\rho \vec{u}(\vec{x}, t)=\int f(\vec{x}, \vec{v}, t) \vec{v} d \vec{v}$. The large-scale limit (Chapman-Enskog expansion) of this equation shows that the moments of the above equation obey the Navier-Stokes equations of continuum fluid mechanics, with a kinematic viscosity $\nu=c_{s}^{2} \tau$, where $c_{s}$ is the sound speed and this information is implicitly provided by the definition of the local equilibrium ([13]).

The lattice Boltzmann equation (LBE) is readily obtained by integrating eq. (1) along the characteristics $\vec{v}=\vec{c}_{i}$, defined by a suitable set of discrete speeds $\vec{c}_{i}, i=0, b$ (for the present study we refer to the standard two-dimensional nine-speed lattice). The resulting equation reads as follows:

$$
f_{i}\left(\vec{x}+\vec{c}_{i} \Delta t, t+\Delta t\right)=f_{i}(\vec{x}, t)+\omega \Delta t\left(f_{i}^{e q}-f_{i}\right) .
$$

In the large-scale limit, the equation (2) can be shown to recover the incompressible Navier-Stokes equations, with a viscosity coefficient $\nu=c_{s}^{2}\left(\tau-\frac{\Delta t}{2}\right)$. The shift in the viscosityrelaxation relation with respect to the $\mathrm{BE}$ is due to the time interval separation between the free-streaming and the particle collision (mathematically, it could be derived through an operator splitting approach as pointed out by [14]). Since the discrete speeds $\vec{c}_{i}$ are constants, 
the left-hand side (free-flight) of equation (2) is exact, a key property for the stability of the method, especially for flows far from equilibrium. On the other hand, the right hand side (collision operator) results from a first-order explicit evaluation of the collision operator. Based on this latter observation, one is led to conclude that LB is only first-order in time.

One of the main observations of this paper is that the lattice Boltzmann equation (LBE), while first-order with respect to the $B E$, is nonetheless a second-order accurate time-marcher for the generalized Boltzmann equation with memory term (BEM as Boltzmann Equation with Memory) $([10,15,16])$ :

$$
\epsilon D^{2} f+D f=\omega\left(f^{e q}-f\right) .
$$

The second order derivative is a memory term, introducing a delay of order $\epsilon$ in the relaxation towards equilibrium. The (singular) limit $\epsilon \rightarrow 0$ recovers the standard Boltzmann equation in relaxation form.

The memory term has been advocated by [15] as a fundamental extension of the Boltzmann equation, acknowledging the fact that the finite-size of the molecules, i.e finite-duration of collision events, introduces first-order effects in the Knudsen number, which cannot be omitted even at the level of the streaming operator. In other words, the continuum Boltzmann equation, as we know it in the form of a first-order hyperbolic equation, should be valid only to first order in the Knudsen number $K n_{\sigma}=\sigma \nabla f / f, \sigma$ being the effective size of the molecule (interaction range). This runs against the widely held view, according to which the Boltzmann equation is valid at all orders in the Knudsen number. It is worth to point out that, due to these finite-size effects, the BEM equation satisfies a generalized continuity equation of the form $\partial_{t} \hat{\rho}+\nabla \cdot \hat{J}=0$, where $\hat{\rho} \equiv \rho+\tau D \rho / D t$ and $\hat{J} \equiv J+\tau D J / D t$. Unlike their point-like counterparts, finite-size particles can be partly in and partly out of the control volume, thus generating fluctuating terms in the continuity equation. Indeed, some authors $[17,18]$ have argued that, because of these fluctuations, the momentum density (fluid momentum per unit volume) and the mass flux rate (mass flow per unit surface and unit time) can no longer be identified, as it is commonly done in fluid dynamics. To the best of our knowledge, this points remains controversial [19], and since it lies beyond the scope of the present work, we shall not deal with it any further. Instead, we note that, with the identification $\tau=d t / 2$, the generalized continuity equation is (to second order in $\tau$ ) still a continuity equation, only evaluated at 
time $t+d t / 2$. Therefore, at least from a computational standpoint, the relation between LB and BEM appears to be well posed. In the following we shall show that the lattice Boltzmann equation (LBE) bears a direct link to a generalized BE with memory.

Prior to proving that LBE is second order accurate with respect to BEM, let us revisit the second-order LBE derived from implicit, Crank-Nicholson time-marching of the BE.

\section{CRANK-NICHOLSON DISCRETISATION OF THE BE}

In the original LB scheme the space-time discretisation of the difference equation (2) is performed through an explicit finite differencing along the particle trajectories (straight lines since the discrete speeds are constant) by taking advantage of an highly simplified Method of Characteristics (MOC). Considering a second-order strategy for the time finite differencing, the lattice Boltzmann equation with BGK approximation reads as follows:

$$
\begin{array}{r}
f_{i}\left(\vec{x}+\vec{c}_{i} \Delta t, t+\Delta t\right)=f_{i}(\vec{x}, t)-\frac{\Delta t}{2 \tau}\left[f_{i}(\vec{x}, t)-f_{i}^{e q}(\vec{x}, t)\right. \\
\left.+f_{i}\left(\vec{x}+\vec{c}_{i} \Delta t, t+\Delta t\right)-f_{i}^{e q}\left(\vec{x}+\vec{c}_{i} \Delta t, t+\Delta t\right)\right] .
\end{array}
$$

Next, in order to provide a unified representation of the various time-marching schemes, let us generalize eq. (4) as follows

$$
\begin{aligned}
& f_{i}\left(\vec{x}+\vec{c}_{i} \Delta t, t+\Delta t\right)=f_{i}(\vec{x}, t) \\
& -\frac{\Delta t}{\tau} \theta\left[f_{i}\left(\vec{x}+\vec{c}_{i} \Delta t, t+\Delta t\right)-f_{i}^{e q}\left(\vec{x}+\vec{c}_{i} \Delta t, t+\Delta t\right)\right] \\
& -\frac{\Delta t}{\tau}(1-\theta)\left[f_{i}(\vec{x}, t)-f_{i}^{e q}(\vec{x}, t)\right],
\end{aligned}
$$

where $\theta$ is a tunable numerical parameter such that $\theta=0$ and $\theta=1 / 2$ correspond to standard LBE and Crank-Nicholson LBE, respectively.

This looks like a daunting implicit equation with simultaneous dependencies, which usually impose the resort to expensive matrix solving procedures. Fortunately, a clever transformation saves the day. By making the following transformation ([1]):

$$
\tilde{f}_{i}=f_{i}+\frac{\Delta t}{2 \tau}\left(f_{i}-f_{i}^{e q}\right)
$$

the implicit equation (4) is turned into an explicit evolution equation again, for the trans- 
formed distribution $\tilde{f}$ :

$$
\tilde{f}_{i}\left(\vec{x}+\vec{c}_{i} \Delta t, t+\Delta t\right)=\tilde{f}_{i}(\vec{x}, t)-\frac{\Delta t}{\tau+\frac{1}{2} \Delta t}\left(\tilde{f}_{i}(\vec{x}, t)-f_{i}^{e q}(\vec{x}, t)\right) .
$$

One may then argue that the price for this implicit to explicit transformation is that retracing the original $f_{i}$ from the transformed $\tilde{f}_{i}$ implies the solution of a local non-linear problem, since $f_{i}^{e q}$ shows a quadratic dependence on $f_{i}$ via the local density and flow speed. However,this difficulty is bypassed, by observing that the transformation

$$
f_{i}=\frac{\tilde{f}_{i}+\frac{\omega \Delta t}{2} f_{i}^{e q}}{1+\omega \Delta t / 2}
$$

implies the following relations for the equilibrium (eq) and non-equilibrium (ne) components, respectively:

$$
\begin{array}{r}
\tilde{f}_{i}^{e q}=f_{i}^{e q}, \\
\tilde{f}_{i}^{n e}=\left(1+\frac{\omega \Delta t}{2}\right) f_{i}^{n e} .
\end{array}
$$

This means that any linear combination of the distribution functions (kinetic moment), $M[f]=M^{e q}+M^{n e}$, obeys the following rules:

$$
\begin{array}{r}
\tilde{M}^{e q}=M^{e q}, \\
\tilde{M}^{n e}=\left(1+\frac{\omega \Delta t}{2}\right) M^{n e} .
\end{array}
$$

The nice consequence is that the the basic hydrodynamic quantities are invariant under the transformation $f \rightarrow \tilde{f}$, i.e., they are obtained through simple summation over $\tilde{f}_{i}$ :

$$
\begin{gathered}
\rho=\sum_{i} f_{i}=\sum_{i} \tilde{f}_{i}, \\
\vec{u}=\sum_{i} \vec{c}_{i} f_{i} / \rho=\sum_{i} \vec{c}_{i} \tilde{f} / \rho .
\end{gathered}
$$

This voids the need of solving the implicit equation (4) to obtain density and hydrodynamic velocity fields. The difference between $f$ and $\tilde{f}$ becomes apparent only at the level of non-conserved quantities, such as the stress tensor and higher-order kinetic moments. Indeed, the stress tensor $S_{a b}$ is related to the non-equilibrium component of the momentum flux tensor, defined through the following local expression:

$$
P_{a b}^{n e}=\sum_{i}\left(c_{i a} c_{i b}-c_{s}^{2} \delta_{a b}\right)\left(f_{i}-f_{i}^{e q}\right) .
$$


where latin indices run over the spatial dimensions. The precise relation to the hydrodynamic strain tensor is as follows:

$$
\tilde{S}_{a b}=-\frac{\omega}{2 \rho c_{s}^{2}} \tilde{P}_{a b}^{n e}=\left(1+\frac{\omega \Delta t}{2}\right) S_{a b}
$$

As long as hydrodynamic moments are conserved, it is therefore clear that a second-order Crank-Nicholson evaluation of the collision operator leads basically to the same algorithm as LB, only for a transformed distribution $\tilde{f}$, with a shifted-rescaled frequency:

$$
\tilde{\omega}=\frac{\omega}{1+\frac{\omega \Delta t}{2}} .
$$

Given that $\tilde{f}$ carries the same density and momentum as the original $f$, whenever the numerical discretization error can be entirely reabsorbed within a time shift of the macroscopic viscosity, it is correctly concluded that the $\tilde{f}$ and $f$ representations are hydrodynamically equivalent, which implicitly shows that LB is second order in time with respect to the BE with a modified relaxation frequency $\tilde{\omega}$.

This derivation is adamant, but nonetheless indirect. As reported in [14], the timestep in the $\mathrm{CN}$ formulation is not restricted by the stability condition $\Delta t<2 \tau$, so that a vanishingly small $\tau$, as required to achieve very small viscosity/high Reynolds numbers, does not necessarily translate to a correspondingly small time-step $\Delta t$. The tradeoff is quite clear: with standard LBE, thanks to the propagation viscosity $\nu_{p}=-c_{s}^{2} \Delta t / 2$, it is possible to achieve vanishing viscosities $\nu=O(\epsilon)$ where $\epsilon \ll 1$, with a non-vanishing time-step $\Delta t=O(\tau)$. In the CN-formulation, on the other hand, vanishing viscosities $\nu=O(\epsilon)$ necessarily imply $\tau=O(\epsilon)$, although this does not necessarily translates into $\Delta t=O(\epsilon)$, since the time step is no longer restricted by stability constraint. Although the two strategies appear to be quite distinct one from another, here they will be shown to be formally and computationally equivalent for the simulation of macroscopic hydrodynamics. Before doing so, we proceed by illustrating a third and independent way to prove second-order accuracy of LBE, which has the merit of dispensing with any implicit time marching procedure, hence no variable transformation.

\section{VERLET DISCRETISATION OF THE BEM}

In the following, we provide a more straightforward demonstration of the second-order accuracy of the LBE. To this end, we recast (3) in the form of a non-linearly driven, damped 
oscillator:

$$
D^{2} f=-\gamma D f+\Omega^{2}\left(f^{e q}-f\right)
$$

where we have set $\gamma=1 / \epsilon$ and $\Omega^{2}=\omega / \epsilon$. Note that standard first order BE is the infinitelydamped limit $\left(\gamma \rightarrow \infty, \Omega \rightarrow \infty, \Omega^{2} / \gamma \rightarrow \omega\right)$ of this second order kinetic equation. In full analogy with Molecular Dynamics, we discretize the above with the popular Verlet scheme ([20]) as follows:

$$
\begin{aligned}
& f_{i}\left(\vec{x}+\vec{c}_{i} \Delta t, t+\Delta t\right)-2 f_{i}(\vec{x}, t)+f_{i}\left(\vec{x}-\vec{c}_{i} \Delta t, t-\Delta t\right) \\
& +\gamma \Delta t / 2\left[f_{i}\left(\vec{x}+\vec{c}_{i} \Delta t, t+\Delta t\right)-f_{i}\left(\vec{x}-\vec{c}_{i} \Delta t, t-\Delta t\right)\right]= \\
& \Omega^{2} \Delta t^{2}\left[f_{i}^{e q}(\vec{x}, t)-f_{i}(\vec{x}, t)\right] .
\end{aligned}
$$

Collecting all terms at the three distinct time levels, we obtain

$$
f\left(\vec{x}+\vec{c}_{i} \Delta t, t+\Delta t\right)=A f_{i}(\vec{x}, t)+B f_{i}\left(\vec{x}-\vec{c}_{i}, t-\Delta t\right)+C f_{i}^{e q},
$$

where

$$
\begin{gathered}
A=\frac{2-\Omega^{2} \Delta t^{2}}{1+\gamma \Delta t / 2}, \\
B=-\frac{1-\gamma \Delta t / 2}{1+\gamma \Delta t / 2}, \\
C=\frac{\Omega^{2} \Delta t^{2}}{1+\gamma \Delta t / 2} .
\end{gathered}
$$

Next, we observe that the standard LBE scheme is a special case of the above, with the choice $A=1-\omega \Delta t, B=0, C=\omega \Delta t$ :

$$
f_{i}\left(\vec{x}+\vec{c}_{i} \Delta t, t+\Delta t\right)=(1-\omega \Delta t) f_{i}(\vec{x}, t)+\omega \Delta t f_{i}^{e q} .
$$

Moreover, it is readily checked that this triple of values is exactly recovered with the choice $\gamma \Delta t=2$, namely $\epsilon=\Delta t / 2$.

The Verlet scheme with dissipative terms is second-order time-accurate, thus proving that $\mathrm{LBE}$ is a second order Verlet time integration of the Boltzmann equation with memory.

\section{RECOVERING BEM FROM CRANK-NICHOLSON LBE}

The circle is now closed by observing that with the choice $\epsilon=\Delta t / 2$, the BEM, given by eq. (3), turns exactly into the second-order Taylor expansion of the standard LBE. 
A second-order Taylor expansion of eq. (7) delivers the following equation:

$$
D_{i} \tilde{f}_{i}+\frac{\Delta t}{2} D_{i}^{2} \tilde{f}_{i}=-\frac{1}{\tau+\frac{1}{2} \Delta t}\left(\tilde{f}_{i}-f_{i}^{e q}\right),
$$

where $D_{i}=\partial_{t}+\vec{c}_{i} \cdot \vec{\nabla}$.

Therefore the Crank-Nicholson evaluation of the collision operator in the discrete LBE is equivalent to a generalized form of the Boltzmann equation, which can be assimilated to the continuum LBE with the addition of a second order memory term $\frac{\Delta t}{2} D_{i}^{2} \tilde{f}_{i}$.

\section{FROM KINETIC THEORY TO FLUID DYNAMICS}

As already pointed out, the moments of BE and BEM both lead to a particular form of the Navier-Stokes equations. Let us call $f, \tilde{f}$ and $g$ the population functions and $\tau_{f}, \tilde{\tau}$ and $\tau_{g}$ the corresponding relaxation parameters for the standard LBE, the LBE formulation with the variable transformation and the LBE formulation derived through the Verlet discretisation of the BEM, respectively.

This leads to the following three kinetic models:

$$
\begin{gathered}
f_{i}\left(\vec{x}+\vec{c}_{i} \Delta t, t+\Delta t\right)=f_{i}(\vec{x}, t)+\frac{\Delta t}{\tau_{f}}\left(f_{i}^{e q}-f_{i}\right), \\
\tilde{f}_{i}\left(\vec{x}+\vec{c}_{i} \Delta t, t+\Delta t\right)=\tilde{f}_{i}(\vec{x}, t)+\frac{\Delta t}{\tilde{\tau}+\Delta t / 2}\left(\tilde{f}_{i}^{e q}-\tilde{f}_{i}\right), \\
g_{i}\left(\vec{x}+\vec{c}_{i} \Delta t, t+\Delta t\right)=g_{i}(\vec{x}, t)+\frac{\Delta t}{\tau_{g}+\Delta t / 2}\left(g_{i}^{e q}-g\right) .
\end{gathered}
$$

Recovering the Navier-Stokes description of a fluid with viscosity $\nu$ through a ChapmanEnskog expansion we obtain:

$$
\begin{gathered}
\tau_{f}=\frac{\nu}{c_{s}^{2}}+\frac{\Delta t}{2}, \\
\tilde{\tau}=\frac{\nu}{c_{s}^{2}}, \\
\tau_{g}=\frac{\nu}{c_{s}^{2}} .
\end{gathered}
$$

Therefore, the simulation of a fluid with viscosity $\nu$ through the three kinetic models requires solving the following equations:

$$
f_{i}\left(\vec{x}+\vec{c}_{i} \Delta t, t+\Delta t\right)=f_{i}(\vec{x}, t)+\frac{\Delta t}{\nu / c_{s}^{2}+\Delta t / 2}\left(f_{i}^{e q}-f_{i}\right)
$$




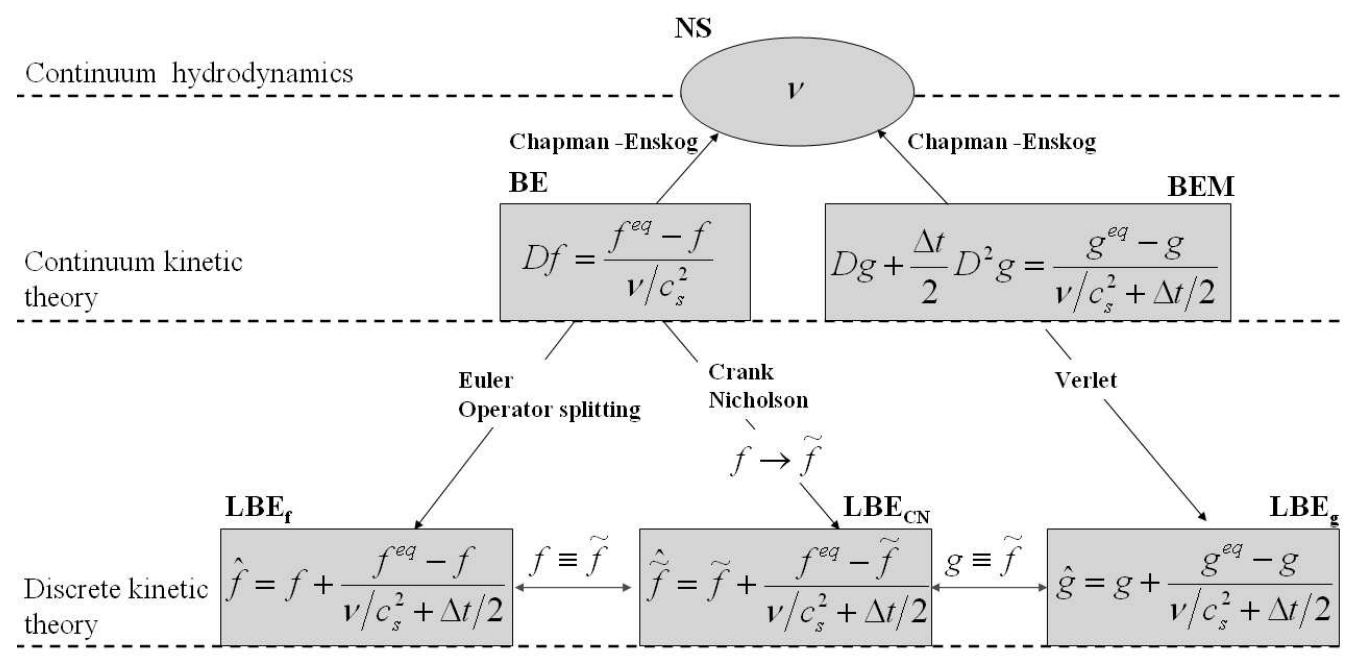

FIG. 1: Schematic diagram of the conceptual links between the three equivalent LB formulations for a continuum fluid with viscosity $\nu$. The diagram straddles across three logical layers: continuum hydrodynamics, continuum kinetic theory and discrete-kinetic theory.

$$
\begin{aligned}
\tilde{f}_{i}\left(\vec{x}+\vec{c}_{i} \Delta t, t+\Delta t\right) & =\tilde{f}_{i}(\vec{x}, t)+\frac{\Delta t}{\nu / c_{s}^{2}+\Delta t / 2}\left(\tilde{f}_{i}^{e q}-\tilde{f}_{i}\right), \\
g_{i}\left(\vec{x}+\vec{c}_{i} \Delta t, t+\Delta t\right) & =g_{i}(\vec{x}, t)+\frac{\Delta t}{\nu / c_{s}^{2}+\Delta t / 2}\left(g_{i}^{e q}-g\right) .
\end{aligned}
$$

This point, summarized in figure 1, clearly demonstrates that, as long as the hydrodynamic moments are conserved, the three schemes are equivalent in terms of macroscopic behaviour in the bulk.

We wish to emphasize that, despite its tight relation to the time-marching procedure, the equivalence between the three kinetic schemes does not imply any statement regarding their accuracy with respect to the hydrodynamic equations. The latter issue is still controversial, not least because of its high sensitivity to the details of the procedures adopted to implement the boundary conditions. In the following section we shall revisit this important issue.

\section{CONVERGENCE AND ACCURACY}

From the numerical point of view, it is not convenient to implement the LB algorithm in the form reported in Eq. (2), because the latter requires non-homogeneous discretization with regards to space and time. It is easy to overcome this problem by rewriting the left hand side of Eq. (2) as: 


$$
f_{i}\left(\vec{x}+\vec{c}_{i} \Delta t, t+\Delta t\right)=f_{i}\left(\vec{x}^{\prime}+\vec{c}_{i}^{\prime}, t^{\prime}+1\right)
$$

where $\vec{x}^{\prime}=\vec{x} / \Delta x, \vec{c}_{i}^{\prime}=\vec{c}_{i} / c, c=\Delta x / \Delta t$ and $t^{\prime}=t / \Delta t$. The non-zero components of the discrete velocities of the nine-speed lattice considered here, all have the same magnitude. If one takes the magnitude of the non-zero components equal to $c$, then the previous expression implies that $\Delta x^{\prime}=\Delta t^{\prime}=1$. The latter assumption is standard in the LBE literature. Designing a lattice such that $\Delta x^{\prime}=\Delta t^{\prime}=1$ allows one to move along the characteristics on a regular mesh, but it does not provide any constraint on $\Delta t$ and $\Delta x$ in physical units.

Let us define $c_{s}$ the lattice sound speed: on the nine-velocity lattice, the following relation holds $c_{s}^{2}=c^{2} / 3$. If $\Delta t \sim \tau$, then $\Delta x \sim c \Delta t \sim \lambda$, where $\lambda$ is the particle mean free-path. Let us define $L$ as the characteristic macroscopic length, so that $k=\Delta x / L \ll 1$ becomes essentially the numerical Knudsen number, and further introduce $U$ as the characteristic macroscopic velocity. Consequently $\Delta t / T=k m$, where $m=U / c$ is proportional to the numerical Mach number and $T=L / U$ is a typical macroscopic time-scale. Hence the parameter $m$ can be expressed equivalently as

$$
m=\frac{\Delta t / T}{\Delta x / L}
$$

Now we can formulate properly the problem of the scaling, which consists in setting the parameter $m$ on a given mesh, i.e. for a given $k$, namely in assuming the function $m(k)$. Let us restrict ourselves to the function $m(k) \sim k^{\alpha}$, where $\alpha$ is an integer. Two strategies are widely used in literature:

- $\alpha=0$, i.e. $m \sim 1$, the so-called advective (or hyperbolic, or acoustic) scaling;

- $\alpha=1$, i.e. $m \sim k$, the so-called diffusive (or parabolic) scaling.

Let us consider first the ideal case, where $\nu=c_{s}^{2} \tau$. In this case, the physical meaning of the discussed scaling strategies can be clarified by introducing the following relation

$$
m=\frac{c_{s}^{2}}{c^{2}} \frac{R e k}{\beta}
$$

where $\beta=\omega \Delta t=\Delta t / \tau$ and $R e=U L / \nu$ is the Reynolds number, or equivalently

$$
M a=R e K n,
$$


where $M a=U / c_{s}$ and $K n=c_{s} \tau / L$ are the Mach and Knudsen's numbers, respectively. The latter relation is equivalent to the so-called von Karman relation. In the diffusive scaling, $\Delta x^{2} / \Delta t=L^{2} / T, m \sim k$ and $\beta=\Delta t / \tau \sim 1$. Hence taking into account Eq. (36) Re is kept fixed. In the acoustic scaling, $\Delta x / \Delta t=L / T, m \sim 1$ and $\beta=\Delta t / \tau \sim k$. In this case, Eq. (36) implies again that $R e$ is kept fixed. The conclusion is that, as far as the ideal case with $\nu=c_{s}^{2} \tau$ is concerned, both the diffusive and the acoustic scaling imply that the Reynolds number is kept fixed.

Unfortunately, in the numerical scheme for $\theta \neq 1 / 2$, the viscosity picks up a $O(\Delta t)$ correction, according to the expressions (38)

$$
\nu=\frac{1}{3} \frac{\Delta x^{2}}{\Delta t^{2}}[\tau+(\theta-1 / 2) \Delta t]
$$

and, equivalenty,

$$
\tau=3 \nu \frac{\Delta t^{2}}{\Delta x^{2}}+(1 / 2-\theta) \Delta t
$$

The previous correction modifies the expression given by Eq. (36), because a different factor $\tilde{\beta}$ must be considered there, where $\tilde{\beta}$ is defined as

$$
\tilde{\beta}(\beta, \theta)=\frac{\beta}{1-(1 / 2-\theta) \beta}
$$

Consequently also Eq. (37) must be modified, namely

$$
M a=\operatorname{Re} K n \beta / \tilde{\beta}
$$

The scaling of the physical and dimensionless numbers upon doubling the grid resolution is summarized in table I, for both diffusive and acoustic scalings. As it is evident from the table, in this case, as far as $\theta \neq 1 / 2$, the diffusive scaling ensures that the Reynolds number is kept fixed, while the acoustic scaling implies some small variation.

Since the diffusive scaling keeps fixed the macroscopic viscosity, it is appropriate for assessing the accuracy of the model towards the macroscopic equations. On the other hand, the advective scaling keeps constant the microscopic relaxation time and hence it is appropriate for the convergence towards continuum kinetic equations. In the following, convergence and accuracy properties are investigated within both scaling regimes. 
TABLE I: The scaling of the physical quantities and dimensionless numbers upon doubling the grid resolution, $d x \rightarrow \Delta x / 2$. In the above $L$ and $T$ denote the typical macroscopic length and time scale of the problem.

\begin{tabular}{r|r|r}
\hline \hline Physical scaling & MICRO LB scaling & MACRO LB scaling \\
\hline \hline$T \rightarrow T$ & Acoustic scaling & Diffusive scaling \\
\hline$L \rightarrow L$ & $T \rightarrow T$ & $T \rightarrow T$ \\
\hline$c_{s} \rightarrow c_{s}$ & $L \rightarrow L$ & $L \rightarrow L$ \\
\hline$U \rightarrow U$ & $c_{s} \rightarrow c_{s}$ & $U \rightarrow U$ \\
\hline$\tau=\nu / c_{s}^{2} \rightarrow \tau$ & $U \rightarrow 2 c_{s}$ \\
$\nu=c_{s}^{2} \tau \rightarrow \nu$ & $\nu=c_{s}^{2}[\tau-(1 / 2-\theta) \Delta t] \rightarrow+O(\Delta t)$ & $U \rightarrow U$ \\
\hline$M a \rightarrow M a$ & $M a \rightarrow M a$ & $\tau=\nu / c_{s}^{2}+(1 / 2-\theta) \Delta t \rightarrow \tau / 4$ \\
$K n \rightarrow K n$ & $K n \rightarrow K n$ & $\nu \rightarrow \nu$ \\
\hline$R e \rightarrow R e$ & $R e \rightarrow R e+O(\Delta t)$ & $M a \rightarrow M a / 2$ \\
& $k \equiv \Delta x / L \rightarrow h / 2$ & $K n \rightarrow K n / 2$ \\
& $\beta \equiv \Delta t / \tau \rightarrow \beta / 2$ & $R e \rightarrow R e$ \\
\hline \hline
\end{tabular}

NUMERICAL VALIDATION

In this section we demonstrate the equivalence of the three formulations through numerical simulation of both laminar and non-laminar flows.

\section{Poiseuille flow}

We begin by considering a linear case, a laminar flow between two parallel plates, driven by a constant acceleration $\vec{a}$, oriented along the $x$-axis and tuned in such a way as to recover the desired Reynolds number Re (i.e. Poiseuille flow).

Periodic boundary conditions are imposed at the inlet and the outlet sections of the computational domain, while no-slip boundary conditions are enforced at the wall through bounce-back reflection of the discrete populations.

In order to impose the desired acceleration $\vec{a}$, let us introduce a modified macroscopic 
velocity $\vec{u}^{*} \neq \vec{u}$ and a new equilibrium distribution function $f_{i}^{e q *}$ such that

$$
\begin{gathered}
\rho=\sum_{i} f_{i}=\sum_{i} f_{i}^{e q *}, \\
\vec{u}=\sum_{i} \vec{c}_{i} f_{i} / \rho \neq \vec{u}^{*}=\sum_{i} \vec{c}_{i} f_{i}^{e q *} / \rho:=\vec{u}+\Delta \vec{u},
\end{gathered}
$$

where $\Delta \vec{u}=\vec{u}^{*}-\vec{u}:=\tau \vec{a}$. Clearly, equations $(6,7,8)$ are still valid, provided that $f_{i}^{e q *}$ is considered instead of $f_{i}^{e q}$. The new equilibrium $f_{i}^{e q *}$ can now be computed from $\tilde{f}_{i}$ by using equation (6), which yields

$$
\vec{u}=\sum_{i} \vec{c}_{i} \tilde{f}_{i} / \rho+\frac{\Delta t}{2} \vec{a} .
$$

Periodic boundary conditions do not raise any problem, while wall boundary conditions require some care. It is known that the bounce-back rule for $f_{i}$ is sufficient to recover no-slip boundary condition half-cell away from the given boundary node. For a computational node close to the wall, bounce-back is equivalent to assume that

$$
f_{\bar{i}}=f_{i}
$$

where $f_{\vec{i}}$ is the distribution function corresponding to the discrete velocity $\vec{c}_{\dot{i}}$, and $\vec{c}_{i}:=-\vec{c}_{i}$ is an incoming discrete particle velocity with respect to the wall. In the case of Crank-Nicholson LBE, the condition given by equation (45) must be reformulated for the populations $\tilde{f}_{i}$, in general by means of the transformation (6). This general case will be discussed in the next numerical test, since for the Poiseuille flow, we can safely take $\tilde{f}_{\bar{i}}=\tilde{f}_{i}$, because the acceleration is orthogonal to the wall surface unit vector.

The results of the numerical computations for different Reynolds numbers ranging between 20 and 22,000 are reported in Table II, which compares the numerical and the analytical values of the viscosity. It is observed that, by tuning the relaxation parameters as suggested in the previous section, identical results (up to the machine precision) are obtained, for a given Reynolds number. This provides a numerical validation of the aforementioned conceptual and computational equivalence between the LBE and the Crank-Nicholson LBE schemes for the macroscopic quantities.

\section{Flow across an array of thin plates}

Strictly speaking, the aforementioned numerical equivalence only applies to the bulk properties, because, in the presence of solid boundaries, transforming condition (45), by 
TABLE II: The error in the numerical viscosity $\nu$ for the Poiseuille flow solved by standard forward Euler (LBE) and by Crank-Nicholson (CN-LBE) at different Re. The number of mesh points along the direction transversal to the flow is $N_{y}=30$. Periodic boundary conditions are set at inlet and outlet and Bounce-back boundary conditions at the wall. The viscosity $\nu$ is expressed in lattice units.

\begin{tabular}{r|ccc|cc}
\hline \hline & \multicolumn{3}{|c|}{$\nu$} & \multicolumn{2}{c}{ Error [\%] on $\nu$} \\
Re & exact & LBE & CN-LBE & LBE & CN-LBE \\
\hline \hline 20 & 0.333333 & 0.331359 & 0.331359 & -0.592182 & -0.592182 \\
40 & 0.166666 & 0.166781 & 0.166781 & 0.069016 & 0.069016 \\
141 & 0.047619 & 0.047749 & 0.047749 & 0.273200 & 0.273200 \\
404 & 0.016666 & 0.016714 & 0.016714 & 0.289161 & 0.289161 \\
1,090 & 0.006172 & 0.006190 & 0.006190 & 0.291084 & 0.291084 \\
2,988 & 0.002252 & 0.002258 & 0.002258 & 0.291349 & 0.291349 \\
8,137 & 0.000827 & 0.000829 & 0.000829 & 0.291385 & 0.291385 \\
22,130 & 0.000304 & 0.000305 & 0.000305 & 0.291389 & 0.291389 \\
\hline \hline
\end{tabular}

means of the mapping (6), is in general a non trivial task. Indeed, by recalling eq. (8) and eq. (45) in terms of $\tilde{f}_{i}$ we obtain

$$
\tilde{f}_{\bar{i}}+\frac{\omega \Delta t}{2} f_{\bar{i}}^{e q *}=\tilde{f}_{i}+\frac{\omega \Delta t}{2} f_{i}^{e q *}
$$

Upon defining $F_{i}(\vec{a})$ such that $\sum_{i} \vec{c}_{i} F_{i}(\vec{a})=\rho \tau \vec{a}$ is the only non-zero moment, it follows that $f_{i}^{e q *}=f_{i}^{e q}+F_{i}$. By introducing the latter expression in equation (46), we obtain

$$
\tilde{f}_{\bar{i}}=\tilde{f}_{i}+\frac{\omega \Delta t}{2}\left(F_{i}-F_{\bar{i}}\right)+\frac{\omega \Delta t}{2}\left(f_{i}^{e q}-f_{\bar{i}}^{e q}\right) .
$$

As compared to the equation (46), two (pairs of) additional terms make their appearance. In particular, due to definition of the equilibrium, the rightmost term leads to an implicit boundary condition. In order to circumvent this difficulty, the following simplification is proposed

$$
\tilde{f}_{\bar{i}}=\tilde{f}_{i}+\frac{\omega \Delta t}{2}\left(F_{i}-F_{\bar{i}}\right)
$$

which clearly amounts to neglect the deficit between the two specular equilibria $f_{i}^{e q}$ and $f_{\bar{i}}^{e q}$. 


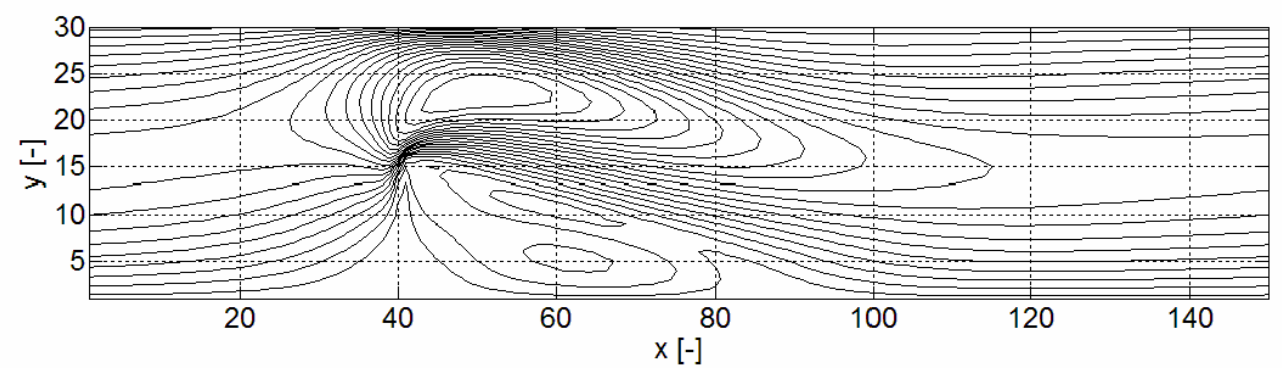

FIG. 2: Iso-contours of the velocity magnitude for a laminar flow between parallel plates with orthogonal screens periodically inter-spaced. The computational domain repeats periodically along the $x$-axis and the screen is located at $4 / 15$ of the total length. The mesh size is $N_{x}=150 \times N_{y}=30$.

In order to inspect the effects of the previous simplification, we next consider a non-linear test case, consisting of a laminar flow between two parallel plates, driven by a constant acceleration $\vec{a}$. Due to inlet/outlet periodicity, this corresponds to an array of periodically spaced orthogonal screens. The computational domain is reported in figure 2 and it is periodically repeated along the $x$-axis. The screen is located at $4 / 15$ of the total length and each screen occupies exactly half of the gap between the plates. The computational mesh is $150 \times 30$ and the screen is discretised by $15 \times 1$ computational nodes. In the following, two simulations are compared by varying the Reynolds number Re defined as follows:

$$
R e=\frac{v L}{\nu}
$$

where $v$ is the average incoming velocity in the reduced section (i.e. half of the total channel section), $L$ is the characteristic length equal to the reduced section height (i.e. half of the total channel section) and $\nu$ is the fluid kinematic viscosity. In particular, in the following, the simulations parameters (in lattice units) are selected: $a_{x} \in\left\{2 \times 10^{-4}, 1 \times 10^{-4}\right\}, \tau \in$ $\{0.2,0.1\}$ and the total number of time steps $N_{t}$ varies from 25000 to 50000 . The previous choices lead to $R e \in\{37.9,60.3\}$ respectively. Periodic boundary conditions apply at both inlet and outlet. Wall boundary conditions are described by Eq. (48).

The numerical results are reported in Table III, which compares both total kinetic energy and Reynolds number for the different numerical time discretization methods (standard forward Euler - LBE and Crank-Nicholson). At variance with the linear test, a (very) small effect of the simplification given in eq. (48) is appreciated. This effect is due to the implemented boundary conditions, as witnessed by the substantial reduction observed by 
TABLE III: Viscosity, $\nu$, kinetic energy, $E$ and Reynolds number, $R e$, for a laminar flow between parallel plates with orthogonal screens periodically inter-spaced. Standard forward Euler (LBE) and Crank-Nicholson (CN-LBE) are considered. The mesh size is $N_{x}=150 \times N_{y}=30$. Periodic boundary conditions are set at the inlet and the outlet. Boundary conditions at the lateral walls and the screen are given by equation (48). Re is defined by taking the average velocity in the reduced section at $4 / 15$ of the total length (half of the total channel section)

\begin{tabular}{c|cc|cc}
\hline \hline & \multicolumn{2}{|c|}{$E$} & \multicolumn{2}{c}{$R$} \\
$\nu$ & LBE & CN-LBE & LBE & CN-LBE \\
\hline \hline 0.066667 & 24.452503 & 24.452858 & 37.909884 & 37.909669 \\
0.033333 & 17.308966 & 17.309025 & 60.286611 & 60.286565 \\
\hline \hline
\end{tabular}

using eq. (48) instead of eq. (47).

\section{Green-Taylor vortex}

We next investigate the accuracy of the various LB versions for the Green-Taylor vortex. This test case is a two-dimensional, unsteady flow induced by a decaying vortex, which involves non-linear flow with periodic boundary conditions. Besides freeing the comparison from uncertainties due to the implementation of the boundary conditions, this test also provides an analytical solution to compare with. The temporal evolution of the velocity components $u$ and $v$ and of the pressure $p$ are given by:

$$
\begin{aligned}
& u(x, y, t)=-u_{0} \cos (k x) \sin (k y) e^{-2 k^{2} \nu t}, \\
& v(x, y, t)=u_{0} \cos (k y) \sin (k x) e^{-2 k^{2} \nu t}, \\
& p(x, y, t)=-\frac{u_{0}^{2}}{4}[\cos (2 k x)+\cos (2 k y)] e^{-4 k^{2} \nu t}+p_{0},
\end{aligned}
$$

where $k=2 \pi / L$ stands for wavenumber of the vortex, $u_{0}$ is the amplitude and $L$ is the edge of the square domain. In the present simulations, we have taken $k=1$, so that there are four vortices in the computational domain $L \times L$, and $u_{0}=1$ and $p_{0}=0$. 
In the next simulations we inspect the accuracy of the LB scheme as a numerical solver for the macroscopic fluid dynamics equations. To this purpose, we tune the microscopic relaxation time $\tau$ so as to recover the same viscosity $\nu$ on different grids (i.e. different numerical Knudsen numbers $k$ ). The asymptotic analysis of LB schemes shows that the discrepancies between the moment and the fluid dynamic equations can be grouped within two different sets, $O(m k)$ and $O\left(k^{2}\right)$, where $m=U / c$ is the numerical Mach number and $k=\Delta x / L \ll 1$ is the numerical Knudsen number. See Ref. [21] for additional details. Hence the global error $E_{g}$ of the numerical solution can be represented as

$$
E_{g}=\left(c_{t} m+c_{x} k\right) k
$$

where $c_{t}$ and $c_{x}$ are two proper constants. Hence, in order to achieve optimal accuracy, it is expedient to choose $m \sim k$. In this section, we take $m=10 k$, corresponding to the diffusive scaling, i.e. $\Delta t=10 T \Delta x^{2} / L^{2}$ (see previous section for details) and this yields

$$
E_{g}^{d}=\left(10 c_{t}+c_{x}\right) k^{2} \sim O\left(k^{2}\right) \sim O\left((\Delta x / L)^{2}\right) \sim O(\Delta t /(10 T))
$$

Taking into account that $\Delta t=10 T \Delta x^{2} / L^{2}$ in Eq. (39) yields

$$
\tau=\left[3 \nu \frac{\Delta t}{\Delta x^{2}}+(1 / 2-\theta)\right] \Delta t,
$$

which means that the microscopic relaxation time $\tau$ is mesh-dependent. In the following, we use $\nu_{0}=1 \times 10^{-3}, L=2 \pi, R e=6283$ and total physical simulation time $T_{t}=10$. The numerical simulations were performed for a series of grid resolutions, with $N_{x} \in\{64,128,256,512\}$ grid points along each direction. This choice leads to $1.95 \times 10^{-3} \leq k \leq 1.56 \times 10^{-2}$ and consequently $1.95 \times 10^{-2} \leq m \leq 1.56 \times 10^{-1}$.

In figure 3 we show cuts of the velocity and pressure profiles, as computed with the LB scheme with $\theta=0,1 / 2$, and compared with the analytical solution (50) (solid line). No appreciable difference between the two numerical solutions with $\theta=0,1 / 2$ is seen, which further corroborates the statement of equivalence between the three LB formulations (the periodic boundary conditions play no role).

To assess the order of convergence towards a macroscopic limit, in figure 4, we report the error on the kinematic viscosity, $E_{\nu}=\nu_{n}-\nu_{0}$, where $\nu_{n}$ is the numerical value of the 


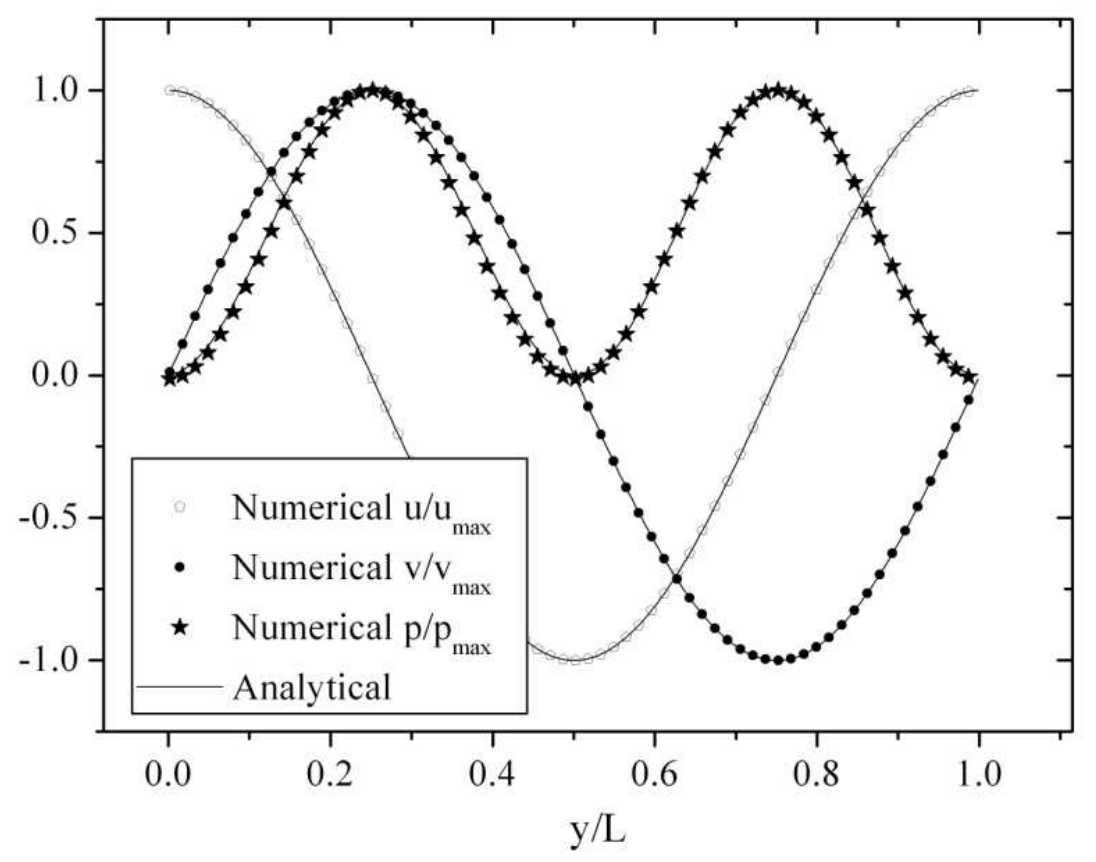

FIG. 3: Cuts of the velocity and pressure profiles as computed with the LB scheme with $\theta=$ $0,1 / 2$, as compared with analytical solution (solid line). No appreciable difference between the two numerical solutions with $\theta=0,1 / 2$ is visible at the scale of the plot.

viscosity calculated through the time decay of the kinetic energy (see equation (50)). From this figure, a very neat second order convergence in the mesh spacing is clearly visible. Since these simulations are performed in the diffusive scaling and $m=10 k$, this corresponds to first order accuracy in the time-step, as already pointed out by Eq. (52).

Once again, we wish to emphasize that all of these results show no appreciable dependence on the value of $\theta$.

\section{Microscopic scaling}

Next, we assess the convergence of the discrete kinetic scheme towards the continuum kinetic limit. The initial conditions are computed assuming the equilibrium distribution with the same fluid dynamic moments used in the previous simulations. Since the equilibrium is a function of the Mach number, in order to ensure the same initialization independently of the mesh resolution, the numerical Mach number was fixed at $m=1 / 5$. This corresponds 


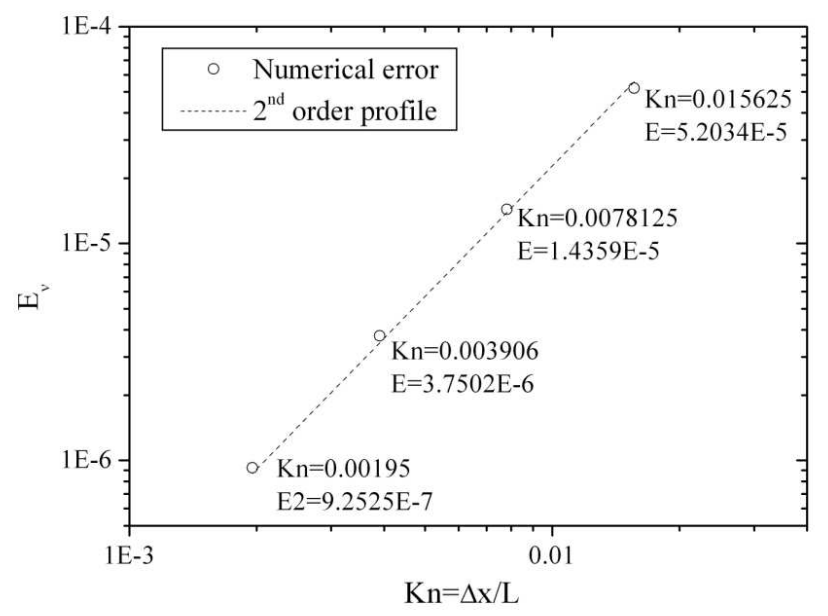

FIG. 4: Error on the kinematic viscosity for the case of the Taylor-Green vortex as a function of the grid resolution. No appreciable difference between the two numerical solutions with $\theta=0,1 / 2$ is visible at the scale of the plot.

to the acoustic scaling, i.e. $\Delta t=T / 5 \Delta x / L$ (see previous section for details) and this yields

$$
E_{g}^{a}=\left(c_{t} / 5+c_{x} k\right) k \sim O(k) \sim O(\Delta x / L) \sim O(5 \Delta t / T)
$$

The previous relation shows that the microscopic scaling is always first order in both space and time with regards to the macroscopic fluid dynamic equations, i.e. for a fixed kinematic viscosity. However it is important to note that, here, the microscopic tuning is focused on the microscopic kinetic equation. The accuracy with regards to the kinetic equation will be discussed next.

Taking into account this condition in Eq. (38) yields

$$
\nu=\frac{1}{3} \frac{\Delta x^{2}}{\Delta t^{2}}[\tau+(\theta-1 / 2) \Delta t]
$$

which means that for $\theta \neq 1 / 2$ the viscosity $\nu$ is mesh dependent. In the following, we used $\tau=0.012, L=2$ and total physical simulation time $T_{t}=0.16$. The numerical simulations were performed on a series of grid resolutions, with $N_{x} \in\{25,50,100,200,400\}$ grid points in each direction. This choice leads to $2.5 \times 10^{-3} \leq k \leq 4.0 \times 10^{-2}$ and consequently $20.9 \leq R e \leq 60.0$. It is worth to point out that the previous choices permits to do away with interpolations both in space and time for comparing the results due to different meshes, i.e. sizes of the computational domain and simulation time are both integer multiple of the 


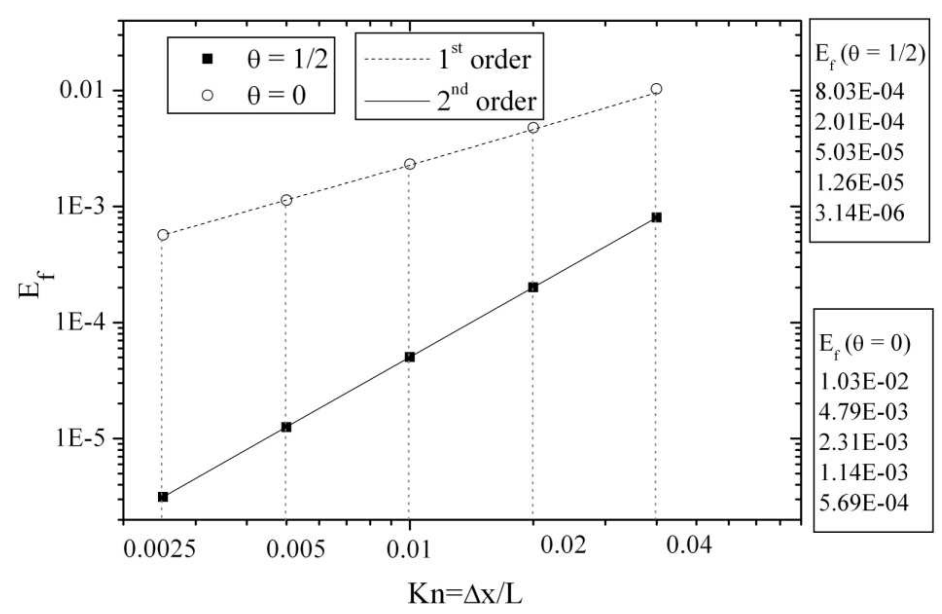

FIG. 5: Error on the discrete distribution function for the case of the Taylor-Green vortex, for $\theta=0$ (open symbols) and $\theta=1 / 2$ (solid squares) at various grid resolutions, $N_{x} \in\{25,50,100,200,400\}$. The numerical Mach number was fixed at $m=1 / 5$, corresponding to numerical Knudsen numbers in the following range $2.5 \times 10^{-3} \leq k \leq 4.0 \times 10^{-2}$

discretization step and time step respectively. This is crucial for a consistent comparison of the numerical results on different grids.

To asses the convergence of the LB model as a kinetic scheme, in figure 5, we monitor the error on the discrete distribution function $E_{f}=\left(\sum_{x, y} \sum_{i}\left(f_{i}(x, y)-f_{i}^{\star}\right)^{2}\right)^{1 / 2}$, where superscript $\star$ refers to the Richardson's extrapolated value. As expected, the case $\theta=1 / 2$ shows a clear second order convergence with the mesh spacing, whereas for $\theta=0$, the convergence is linear. Besides the order of convergence, it is worth noting that the case $\theta=1 / 2$ entails a dramatic drop of the prefactor, as witnessed by the substantial reduction of the error (about two orders of magnitude) even at relatively low-resolution $(k=0.04)$.

The above results refers to $m=0.2$, but we have checked that a similar behaviour is obtained up to $m=0.5$, a value at which the simulation with $\theta=0$ breaks down. This indicates that the time-centered integration does indeed offer significant numerical advantages, both in terms of accuracy and stability, for the discrete kinetic solver versus the continuum kinetic equation. 


\section{BROKEN EQUIVALENCE}

The numerical results presented in the previous section indicate that the equivalence among the different formulations may be (weakly) broken in the presence of non-trivial boundary conditions. This raises the more general issue of broken-equivalence between the three formulations for the case of driven-flows, in which hydrodynamic moments are no longer conserved, as is in the presence of chemical reactions or external forces [22]. Moreover, in case of multi-species flows, although the total momentum of the mixture is conserved, the momentum of the single species is not, because of momentum transfer among the species.

The crucial point is that, once density and/or momentum are no longer conserved, the collision term may 'propagate' the lack of conservation down the lower-order description of the flow, at the Euler level. As a result, unless the numerical discretization is sufficiently accurate, spurious numerical terms may then appear at both Euler and Navier-Stokes level. Under such circumstances, the 'trick' of re-absorbing these spurious terms within 'renormalized' effective transport coefficients, such as the propagation viscosity previously discussed, is no longer viable. In order to clarify this point, let us recall the generalized equilibrium $f_{i}^{e q *}$, as given by equations $(42,43)$. Next, in order to provide a unified representation of the various time-marching schemes, let us consider Eq. (5).

We are interested in analyzing the macroscopic equations recovered in case of diffusive scaling. Let us define $c_{s}$ the lattice sound speed and $c=\Delta x / \Delta t$ the non-zero component of the generic velocity on the nine-velocity lattice (on the latter, the following relation holds $\left.c_{s}^{2}=c^{2} / 3\right)$. Both speeds are representative of the microscopic dynamics on the lattice. If $\Delta t \sim \tau$, then $\Delta x \sim c \Delta t \sim \lambda$, where $\lambda$ is the particle mean free-path. Let us define $L$ as the characteristic macroscopic length, so that $k=\Delta x / L \ll 1$ becomes essentially the Knudsen number, and further introduce $U$ as the characteristic macroscopic velocity. Consequently $\Delta t / T=k(U / c)$, where $U / c$ is proportional to the Mach number and $T=L / U$ is a typical macroscopic time-scale. On the assumption that $U / c \sim k$, so that $\Delta t / T \sim k^{2}$, we can write the left hand side of Eq. (2) as:

$$
f_{i}\left(\vec{x}+\vec{c}_{i} \Delta t, t+\Delta t\right)=f_{i}\left(\vec{x}^{\prime \prime}+k \vec{c}_{i}^{\prime \prime}, t^{\prime \prime}+k^{2}\right)
$$

where $\vec{x}^{\prime \prime}=\vec{x} / L, \vec{c}_{i}^{\prime \prime}=\vec{c}_{i} / c_{s}$ and $t^{\prime \prime}=t / T$. It is worth to point out the difference between the previous expression and the one used in Eq. (34) for the numerical implementation of 
the algorithm. Similar considerations hold for the equilibrium distribution function. By applying the usual Taylor expansion, followed by the Chapman-Enskog procedure using $k$ as a perturbation parameter, it can be shown (see Appendix) that the continuity equation picks up a diffusive contribution proportional to $c_{s}^{2}(\theta-1 / 2)$, thereby proving that local mass conservation is recovered only the case $\theta=1 / 2$. Hence, whenever $\theta \neq 1 / 2$ and one of the hydrodynamic moments is not conserved, lack of conservation reflects into lowerlying moments as well. The Crank-Nicholson integration rule and the ensuing variable transformation circumvents this problem.

A simple example of the broken equivalence is provided by the decay of a sine-wave density profile [23] in multi-component fluid mixture. As is known, the mass diffusion coefficient can be measured either by monitoring the decay rate of the density perturbation, or by inspecting the interspecies mass flow (Fick"s law). Any discrepancy in the two measurements necessarily signals a violation of the continuity equation. As shown in [23], such violation can be tamed by using the Crank-Nicolson method with the variable transformation [24].

\section{CONCLUSIONS}

Summarizing, it is known that LBE, a first-order scheme for the continuum Boltzmann equation with relaxation time $\tau$, becomes second-order for the same equation, with a shifted relaxation $\tau+\Delta t / 2$. This shift permits to achieve vanishing low fluid viscosities $\nu=c_{s}^{2}(\tau-\Delta t / 2)$, without taking the relaxation parameter $\tau$ and the time step $\Delta t$ to correspondingly vanishingly small values, thereby avoiding the time-step collapse and the ensuing dramatic drop in computational efficiency for the simulation of turbulent flows. The implicit Crank-Nicholson formulation is a second-order scheme for the continuum BE with relaxation $\tau$. Since the corresponding fluid viscosity is unshifted, $\nu=c_{s}^{2} \tau$, small viscosities imply correspondingly small values of $\tau$. However, this does not imply any time-step collapse, because implicit formulations are free from the stability constraint $\Delta t<2 \tau$. While the two formulations were so far regarded as distinct lattice kinetic schemes for the numerical simulation of hydrodynamic flows, in this work we have analytically shown and numerically demonstrated, that they are basically equivalent in terms of macroscopic hydrodynamics simulation. In addition, we have shown that the above versions are also equivalent to a Verlet time-marching for a generalized Boltzmann equation with memory delay exactly equal 
to $\Delta t / 2$. This proves second order time accuracy towards the continuum limit, with no need of invoking implicit formulations. This provides an elegant unified picture of the three-fold path to LBE from continuum kinetic equations. We have also emphasized that this equivalence does not imply second order time accuracy towards the hydrodynamic equations. The numerical analysis of time accuracy is performed by simulating a Taylor-Green vortex in a two-dimensional square box. The result is that the implicit Crank-Nicholson formulation is second-order accurate in time to the continuous BE, but only first order (and equivalent to LBE) to the incompressible Navier-Stokes equations in the appropriate (diffusive) limit $\Delta x^{2} \propto \Delta t$. Finally, we have pointed out that the aforementioned equivalence between the three representations breaks down in the case where mass and/or momentum are no longer conserved on a physical basis (external forcing, chemical reactions in fluid mixtures). In this case, the Crank-Nicholson formulation is singled out as the only one securing numerical conservation of the physically conserved quantities. However expensive matrix solving procedures can be avoided by the discussed variable transformation, as already pointed out in Ref. [24-26].

\section{APPENDIX}

Taylor expansion of the equation (56) yields

$$
f_{i}\left(\vec{x}^{\prime \prime}+k \vec{c}_{i}^{\prime \prime}, t^{\prime \prime}+k^{2}\right)=\sum_{j=0}^{\infty} \frac{1}{j !}\left(k \vec{c}_{i}^{\prime \prime} \cdot \vec{\nabla}^{\prime \prime}+k^{2} \frac{\partial}{\partial t^{\prime \prime}}\right)^{j} f_{i}\left(\vec{x}^{\prime \prime}, t^{\prime \prime}\right) .
$$

Next, let us expand the solution in powers of the smallness parameter $k=\Delta x / L$ :

$$
f_{i}=f_{i}^{e q *}+\sum_{m=1}^{\infty} k^{m} f_{i}^{(m)}
$$

Taking the zero-order hydrodynamic moments of the previous expansion and recalling that the result must be valid for any $k \ll 1$, yields

$$
\sum_{i} f_{i}^{(m)}=0, \quad \forall m \geq 1
$$

In the case of the first-order hydrodynamic moment, lack of conservation appears, namely

$$
\sum_{i} \vec{c}_{i}^{\prime \prime}\left(f_{i}-f_{i}^{e q *}\right) / \rho=-k \Delta \vec{u}^{\prime \prime}
$$


If $\Delta \vec{u}^{\prime \prime}=\Delta \vec{u} / U \sim 1$ (i.e. $U$ scales $\Delta \vec{u}$ as well), then substituting the regular expansion in the previous expression yields

$$
\begin{gathered}
\sum_{i} \vec{c}_{i}^{\prime \prime} f_{i}^{(1)} / \rho=-\Delta \vec{u}^{\prime \prime} \\
\sum_{i} \vec{c}_{i}^{\prime \prime} f_{i}^{(m)} / \rho=0, \quad \forall m \geq 2 .
\end{gathered}
$$

Introducing the regular expansion in eq. (5) and grouping together terms of the same order in $k$ yields

$$
\vec{c}_{i}^{\prime \prime} \cdot \vec{\nabla}^{\prime \prime} f_{i}^{e q *}=-\omega f_{i}^{(1)}
$$

Taking the hydrodynamic moments of the previous expression yields

$$
\begin{gathered}
\vec{\nabla}^{\prime \prime} \cdot\left(\rho \vec{u}^{\prime \prime *}\right)=0, \\
\frac{1}{3 \omega} \vec{\nabla}^{\prime \prime} \rho=\rho \Delta \vec{u}^{\prime \prime}=-\rho\left(\vec{u}^{\prime \prime}-\vec{u}^{\prime \prime *}\right),
\end{gathered}
$$

which represent the zero-order approximations of the continuity and momentum equation respectively. Similarly, by introducing the regular expansion in eq. (5) and grouping the terms of the same order in $k^{2}$ yields

$$
\begin{aligned}
& \vec{c}_{i}^{\prime \prime} \cdot \vec{\nabla}^{\prime \prime} f_{i}^{(1)}+\left(\frac{1}{2}\left(\vec{c}_{i}^{\prime \prime} \cdot \vec{\nabla}^{\prime \prime}\right)^{2}+\frac{\partial}{\partial t^{\prime \prime}}\right) f_{i}^{e q *}= \\
& -\omega f_{i}^{(2)}-\omega \theta \vec{c}_{i}^{\prime \prime} \cdot \vec{\nabla}^{\prime \prime} f_{i}^{(1)} .
\end{aligned}
$$

Combining eqs. $(63,66)$ yields

$$
\begin{aligned}
& -\frac{1}{\omega}\left(\vec{c}_{i}^{\prime \prime} \cdot \vec{\nabla}^{\prime \prime}\right)^{2} f_{i}^{e q *}+\left(\frac{1}{2}\left(\vec{c}_{i}^{\prime \prime} \cdot \vec{\nabla}^{\prime \prime}\right)^{2}+\frac{\partial}{\partial t^{\prime \prime}}\right) f_{i}^{e q *}= \\
& -\omega f_{i}^{(2)}+\theta\left(\vec{c}_{i}^{\prime \prime} \cdot \vec{\nabla}^{\prime \prime}\right)^{2} f_{i}^{e q *},
\end{aligned}
$$

and consequently

$$
\frac{\partial f_{i}^{e q *}}{\partial t^{\prime \prime}}=-\omega f_{i}^{(2)}+\left(\frac{1}{\omega}-\frac{1}{2}+\theta\right)\left(\vec{c}_{i}^{\prime \prime} \cdot \vec{\nabla}^{\prime \prime}\right)^{2} f_{i}^{e q *}
$$

Taking the zero-order hydrodynamic moment, we obtain

$$
\frac{\partial \rho}{\partial t^{\prime \prime}}=\frac{1}{3}\left(\frac{1}{\omega}-\frac{1}{2}+\theta\right) \vec{\nabla}^{\prime \prime 2} \rho
$$

which represents the first-order approximation of the continuity equation. Collecting the terms up to $k^{2}$ in the continuity equation given by eqs. $(64,69)$, yields

$$
\frac{\partial \rho}{\partial t^{\prime \prime}}+\vec{\nabla}^{\prime \prime} \cdot\left(\rho \vec{u}^{\prime \prime *}\right)=\frac{1}{3}\left(\frac{1}{\omega}-\frac{1}{2}+\theta\right) \vec{\nabla}^{\prime \prime 2} \rho .
$$


Taking into account eq. (65), delivers

$$
\frac{\partial \rho}{\partial t^{\prime \prime}}+\vec{\nabla}^{\prime \prime} \cdot\left(\rho \vec{u}^{\prime \prime}\right)=\frac{1}{3}\left(\theta-\frac{1}{2}\right) \vec{\nabla}^{\prime 2} \rho,
$$

which shows that, if $\theta \neq 1 / 2$, then the continuity equation is not satisfied, i.e. the zero-th order hydrodynamic moment $\rho$ inherits non-conservation from the first-order hydrodynamic moment $\vec{u}$. Further details can be found in [24].

\section{REFERENCES}

[1] X. He, S. Chen, and G. D. Doolen, J. Comput. Phys. 146, 282 (1998).

[2] R. Benzi, S. Succi, and M. Vergassola, Phys. Rep. 222, 145 (1992).

[3] F. Higuera, S. Succi, and R. Benzi, Europhys. Lett. 9, 345 (1989).

[4] G. Mc Namara and L. Zanetti, Phys. Rev. Lett. 61, 2332 (1988).

[5] H. Chen, S. Chen, and W. Mattheus, Phys. Rev A 45, 5339 (1992).

[6] Y. Li, R. Shock, R. Zhang, and H. Chen, Journal of Fluid Mechanics 519, 273 (2004).

[7] S. Succi and R. Benzi, Physica D 69, 327 (1993).

[8] Z. Guo and T. Zhao, Phys. Rev. E 67 (2003).

[9] A. Bardow, I. V. Karlin, and A. A. Gusev, Europhys. Lett. 75, 434 (2006).

[10] S. Ubertini and S. Succi, Commun. Comput. Phys. 3, 342 (2008).

[11] Y. Qian, D. d'Humieres, and P. Lallemand, Europhys. Lett. 17, 479 (1992).

[12] S. Succi, The Lattice Boltzmann Equation for Fluid Dynamics and Beyond (Oxford Clarendon, 2001).

[13] D. Wolfe-Gladrow, Lattice Gas Cellular Automata and Lattice Boltzmann Model (Springer Verlag, 2000).

[14] K. Xu and X. He, Journal of Computational Physics 190, 100 (2003).

[15] B. V. Alexeev, Generalized Boltzmann Physical Kinetics (Elsevier, 1996).

[16] S. Ubertini, G. Bella, and S. Succi, Mathematics and Computers in Simulation 72, 237 (2006).

[17] H. Oettinger, Phys. Rev. Lett. 99 (2007).

[18] H. Brenner, Phys. Rev. E 70 (2004). 
[19] M. Liu, Phys Rev. Lett. 5535 (2008).

[20] D. Rapaport, The Art of Molecular Dynamics Simulation (Cambridge University Press, 2004).

[21] P. Asinari and T. Ohwada, Comput. Math. Appl. 58, 841 (2009).

[22] M. Sbragaglia, R. Benzi, L. Biferale, X. Shan, H. Chen, and S. Succi, J. Fluid Mechanics 628, 299 (2009).

[23] P. Asinari, Phys. Rev. E 73 (2006).

[24] P. Asinari, Phys. Rev. E 77 (2008).

[25] S. Arcidiacono, J. Mantzaras, S. Ansumali, I. Karlin, and C. Frouzakis, Phys. Rev. E 74 (2006).

[26] S. Arcidiacono, I. Karlin, J. Mantzaras, and C. Frouzakis, Phys. Rev. E 76 (2007). 